\title{
Polytopic Model Based Interaction Control for Soft Tissue Manipulation
}

\author{
Árpád Takács*§, József Kuti ${ }^{\dagger \ddagger}$, Tamás Haidegger*§, Péter Galambos*, Imre J. Rudas* \\ *Antal Bejczy Center for Intelligent Robotics, Óbuda University, Bécsi út 96/b, 1034 Budapest, Hungary \\ ${ }^{\dagger}$ Dept. of Telecommunication \& Media Informatics, Budapest University of Technology and Economics, \\ Magyar Tudósok Krt. 2., 1117 Budapest, Hungary \\ $\ddagger$ Institute for Computer Science and Control, Hungarian Academy of Sciences, Kende u. 13-17, 1111 Budapest, Hungary \\ $\S$ Austrian Center for Medical Innovation and Technology, Viktor-Kaplan-Strasse 2, 2700 Wiener Neustadt, Austria \\ E-mail: \{arpad.takacs, haidegger, peter.galambos, imre.rudas\}@irob.uni-obuda.hu, kuti@sztaki.mta.hu
}

\begin{abstract}
Reliable force control is one of the key components of modern robotic teleoperation. The performance of these systems in terms of safety and stability largely depends on the controller design, as it is desired to account for various disturbing conditions, such as uncertainties of the model parameters or latency-induced problems. This work presents a polytopic qLPV model derived from a previously verified nonlinear soft tissue model, along with a model-based force control scheme that involves a tensor product polytopic state feedback controller. The derivation is based on the Tensor Product (TP) Model Transformation. The proposed force control scheme is verified and evaluated through numerical simulations.
\end{abstract}

Index Terms-Soft tissue modeling, telesurgery control, Polytopic model based control, TP Model Transformation, qLPV modeling, LMI-based controller design

\section{INTRODUCTION}

Surgical robotics is one of the most rapidly developing fields in robotics, representing a fine example of HumanMachine Interfaces (HMI) [1]. While many surgical gestures have already been implemented with a degree of autonomy, most of these devices are still used as teleoperation systems. This means that a human surgeon as an operator is always required to be present in the control loop. Modern telesurgical systems dominantly use only visual feedback, while the applicability of force or haptic feedback has been a recurring research topic in the field [2]. Force control incorporating haptic feedback is a widely discussed problem in master-slave teleoperation systems, an efficient implementation can enhance the surgeon's sensory capabilities during the operation. On the other hand, long distance teleoperation inherently carries the difficulties caused by latency (time-delay due to the signal transfer and processing). This phenomenon can lead to stability issues in force-controlled teleoperation systems, which mostly occurs in the case of contact with hard surfaces.

The solution to these issues have been in the focus of many research in the field of control theory [3]. Some of the most promising approaches address these problems through modelbased control. These methods require a reliable mathematical model for the representation of the manipulated substance, or the human tissue. While in most cases the hard tissue, such as bone can be considered as rigid body, creating an accurate model of soft tissue (organs muscles, or the skin) is a complex problem. Today, a large variety of tool-tissue interaction models for surgical robotics applications exist [4].

\section{RELATED WORK}

The problem of reaction force estimation and force control in surgical robotics can be approached from various angles. No general ideal solution exists due to the complexity of the instruments, wide range of required control methods and limitations in the final applications (such as sterilization or restrictions on sensor placement and mounting). One of the first architectures of such control was developed for ROBODOC, the first robotic system to perform complete hip replacement [5]. The control algorithm provided an intuitive HMI allowing the surgeon to guide the robot in a collaborative manner, while force feedback was used to modify the feed rate for cutting, achieving a force controlled velocity input. Lee et al. presented a sensorless method for estimating reaction forces acting on a typical surgical robotic instrument, using a state observer. In their approach, they used a sliding mode control with sliding perturbation observer (SMCSPO) for the instrument manipulation [6]. Yuen et al. showed that a force control method using feed-forward motion terms can largely improve the force tracking performance in the case of contact with soft tissues, which is a crucial problem for manipulating loosely attached or moving organs e.g. during beating heart surgery [7]. Another relevant work in the topic of force tracking in beating heart surgery was published by Liu et al., utilizing the Kelvin-Boltzmann viscoelastic model [8]. Moreira et al. introduced a method for soft tissue force control using active observers and a viscoelastic interaction model, confirming that using a realistic tissue model can increase the performance of the force control [9]. Force control has also been an emerging field of interest in robotic catheter cardiac ablation [10] and in minimally invasive surgery [11].

This study extends the previous works of the authors, where a nonlinear soft tissue model was obtained based on the experimental investigation of the force response of beef liver specimens during tool-tissue interaction [12], amended with [13], a polytopic qLPV (quasi Linear Parameter Varying) 
model of the tissue dynamics that is - regarding its mathematical formalism-suitable for direct use of LMI-based control design methods. As a next step, a model-based force control scheme is presented, utilizing an off-the-shelf tooltissue interaction model. The discussed structure involves a model-based controller, where the required states for the statefeedback controller were acquired using a reference dynamic model of the system, derived using the nonlinear model, developed previously [12]. The discussed approach utilizes the Tensor Product Model Transformation (TP transformation for short) [14] as a systematic methodology capable of transforming analytical nonlinear qLPV state-space representations into polytopic form, which can be directly used in LMI-based multi-objective controller synthesis.

\section{TENSOR PRoduct Model TRANSFormation}

The concept of Tensor Product Model Transformation was introduced by Baranyi [15], and a practical guide for its applicability for qLPV control theory was published in [14]. The basic idea behind TP Model Transformation is the transformation of an arbitrary function into polytopic TP form which is also capable of describing nonlinear dynamical systems for the purpose of controller design via linear matrix inequalities.

In this section, some of the fundamental definitions of the Tensor Product Model Transformation are recalled.

Definition 1: (LPV/qLPV model): Consider the following Linear Parameter Varying model:

$$
\left[\begin{array}{l}
\dot{\mathbf{x}}(t) \\
\mathbf{y}(t) \\
\mathbf{z}(t)
\end{array}\right]=\mathbf{S}(\mathbf{p}(t))\left[\begin{array}{c}
\mathbf{x}(t) \\
\mathbf{u}(t) \\
\mathbf{w}(t)
\end{array}\right]
$$

with state vector $\mathbf{x}(t)$, measured output $\mathbf{y}(t)$, performance output $\mathbf{z}(t)$, input $\mathbf{u}(t)$, and disturbance input $\mathbf{w}(t)$. The $\mathbf{S}(\mathbf{p}(t)) \in \mathbb{S}$ system matrix can be partitioned to $\mathbf{A}(\mathbf{p}(t))$, $\mathbf{B}(\mathbf{p}(t)), \mathbf{C}(\mathbf{p}(t))$, etc. system matrices, and it is defined over a hyper-rectangular parameter domain:

$$
\mathbf{p}(t) \in \Omega=\left[a_{1}, b_{1}\right] \times\left[a_{2}, b_{2}\right] \times \ldots \times\left[a_{N}, b_{N}\right] \subset \mathbb{R}^{N} .
$$

If the parameters in $\mathbf{p}(t)$ are not independent from the $\mathbf{x}(t)$ state variables, it is called quasi-LPV (qLPV) model.

Definition 2: (Finite element polytopic model): The (1) LPV/qLPV model, where the system matrix is given as convex combinations of vertex system matrices, as

$$
\mathbf{S}(\mathbf{p})=\sum_{r=1}^{R} w_{r}(\mathbf{p}) \mathbf{S}_{r} \quad \forall \mathbf{p} \in \Omega,
$$

where

$$
\sum_{r=1}^{R} w_{r}(\mathbf{p})=1, \quad w_{r}(\mathbf{p}) \geq 0 \quad \forall r, \mathbf{p} \in \Omega .
$$

The term finite indicates that $R$ is bounded.

Definition 3: (Finite element polytopic TP model): The (1) LPV/qLPV model, where the system matrix is given as convex combinations of vertex system matrices, and the weighting functions are decomposed to product of univariate functions:

$$
\mathbf{S}(\mathbf{p}(t))=\sum_{j_{1}=1}^{J_{1}} \sum_{j_{2}=1}^{J_{2}} . . \sum_{j_{N}=1}^{J_{N}} \prod_{n=1}^{N} w_{j_{n}}^{(n)}\left(p_{n}(t)\right) \mathbf{S}_{j_{1}, j_{2}, . ., j_{N}} .
$$

Applying the compact notation based on tensor algebra (Lathauwer's work [16]) one has:

$$
\mathbf{S}(\mathbf{p}(t))=S \underset{n=1}{\bigotimes_{n}} \mathbf{w}^{(n)}\left(p_{n}(t)\right),
$$

where the core tensor $\mathcal{S} \in \mathbb{S}^{J_{1} \times J_{2} \times \cdots \times J_{N}}$ is constructed from the vertex system matrices $\mathbf{S}_{j_{1}, j_{2}, \ldots, j_{N}} \in \mathbb{S}$, and the row vector $\mathbf{w}^{(n)}\left(p_{n}(t)\right)$ contains scalar weighting functions $w_{j_{n}}^{(n)}\left(p_{n}(t)\right)$, $\left(j_{n}=1 \ldots J_{N}\right)$, that represents convex combinations as (4) for all $n$.

Remark 1: The polytopic TP model (6) is a special class of polytopic models, where the weighting functions are decomposed to the tensor product of univariate functions.

Definition 4: (TP Model Transformation): TP Model Transformation is a numerical method that transforms the LPV/qLPV models to polytopic TP model, so that the LMI methods developed for polytopic model based control can be applied to the resulting model [15].

The polytopic TP representation of an LPV/qLPV system can be obtained in various ways, of which the MVS-type polytopic model is used in this work, defined below:

Definition 5 (MVS Polytopic TP model): The (6) polytopic TP model, where the $\mathcal{S} \in \mathbb{S}^{J_{1} \times \cdots \times J_{N}}$ core tensor is constructed from the $\mathbf{S}_{j_{1}, \ldots, j_{N}}$ matrices, in such a way that the $(\mathcal{S})_{j_{n}=j} n$-mode subtensors construct the minimal volume enclosing simplex for the

$$
\mathcal{S} \times_{n} \mathbf{w}_{j_{n}}^{(n)}\left(p_{n}\right)
$$

trajectory for all $n=1 \ldots N$.

In the proposed structure, a TP-type polytopic controller is utilized, where the control signal is computed as:

$$
\mathbf{u}=-\left(\mathcal{F} \bigotimes_{n=1}^{N} \mathbf{w}^{(n)}\left(p_{n}(t)\right)\right) \mathbf{x} .
$$

Feedback gains $\mathbf{F}_{i_{1}, i_{2}, \ldots, i_{N}}$ are stored in tensor $\mathcal{F}$.

It is important to note that the discussed model representations are also valid in discrete time domain, with no fundamental restrictions. Further reading about the TP Model Transformation, the MVS-type polytopic TP model generation and manipulation methods can be found in [17], [18], [19], [20].

\section{Nonlinear Soft Tissue Model}

Soft tissue models have been widely discussed in recent years, in order to enhance the performance of common surgical robotic interventions, such as cutting, indentation or grabbing [21]. A reliable tool-tissue interaction model is crucial to achieve high precision force control, and the successful implementation of haptic tools into telesurgical systems [22]. One of the most popular soft tissue model families are 


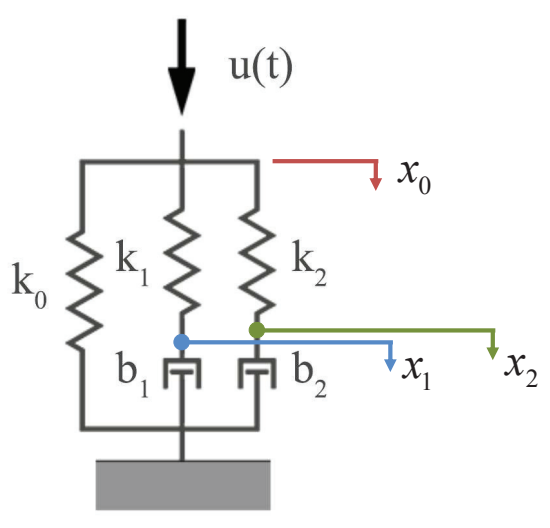

Fig. 1. The Wiechert model of viscoelasticity.

the mass-spring-damper tissue models, often referred to as rheological soft tissue models. Force relaxation data from various indentation experiments is often used for the validation of these models [23], [24]. Yamamoto investigated the applicability of the most commonly used rheological models in the case of point-to-point palpation [25]. Troyer et al. created a hybrid rheological model for finite element modeling implementation [26], while a nonlinear model approach, a variant of the nonlinear Wiechert model was proposed and recently validated by Takács et al. in [27]. The schematic figure of the Wiechert model is shown in Fig. 1. This nonlinear model introduces progressive stiffness characteristics as follows:

$$
k_{j}\left(\chi_{j}\right)=K_{j} e^{\kappa_{j} \chi_{j}(t)},
$$

for $j=0,1,2$, where $\chi$ is the elongation of the spring elements, $K_{j}$ and $\kappa_{j}$ are mechanical parameters, which were taken from experimental data through curve fitting. Introducing two virtual mass points $x_{j}, j=1,2$ at the connection of the spring and damper elements, the model has a total of 3 Degrees of Freedom (DoF). The input of the model is $u(t)$ deformation rate, while the output $y(t)$ is the reaction force arising during the compression of the tissue. The following set of differential equations describe the system mechanics.

$$
\begin{gathered}
\dot{x}_{0}(t)=u(t) \\
\dot{x}_{1}(t)=\frac{1}{b_{1}} K_{1}\left(x_{0}(t)-x_{1}(t)\right) e^{\kappa_{1}\left(x_{0}(t)-x_{1}(t)\right)} \\
\dot{x}_{2}(t)=\frac{1}{b_{2}} K_{2}\left(x_{0}(t)-x_{2}(t)\right) e^{\kappa_{2}\left(x_{0}(t)-x_{2}(t)\right)} \\
\begin{aligned}
y(t)=K_{0} x_{0}(t) e^{\kappa_{0} x_{0}(t)}+K_{1}\left(x_{0}(t)-x_{1}(t)\right) e^{\kappa_{1}\left(x_{0}(t)-x_{1}(t)\right)}+ \\
\quad+K_{2}\left(x_{0}(t)-x_{2}(t)\right) e^{\kappa_{2}\left(x_{0}(t)-x_{2}(t)\right)}
\end{aligned}
\end{gathered}
$$

where $b_{i}, i=1,2$ are the linear damping parameters, also acquired from experimental data. The numeric values of the mechanical parameters are listed in Table I.

\section{Polytopic TP Model of the System}

Regarding the Polytopic TP Model of the nonlinear system described in (10) and (11), the detailed derivation of the model can be found in [13], rearranged in a way that considers the socalled error dynamics. The proposed qLPV model assumes that the control goal is the force control of the surgical instrument at the tissue surface contact.

Based on this, the state variables of the system are:

$$
\mathbf{x}(t)=\left[\begin{array}{l}
x_{0}(t) \\
x_{1}(t) \\
x_{2}(t)
\end{array}\right]
$$

while the output of the system is defined by $y(t)$ The general form of the qLPV model is

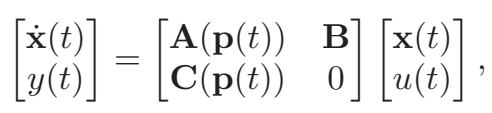

where

$$
\begin{aligned}
\mathbf{p}(t) & =\left[\begin{array}{lll}
e^{\kappa_{1} x_{1}(t)} & e^{\kappa_{2} x_{2}(t)} & e^{\kappa_{0} x_{0}(t)}
\end{array}\right], \\
\mathbf{A}(\mathbf{p}) & =\left[\begin{array}{ccc}
0 & 0 & 0 \\
\frac{K_{1}}{b_{1}} p_{1} & -\frac{K_{1}}{b_{1}} p_{1} & 0 \\
\frac{K_{2}}{b_{2}} p_{2} & 0 & -\frac{K_{2}}{b_{2}} p_{2}
\end{array}\right], \mathbf{B}=\left[\begin{array}{l}
1 \\
0 \\
0
\end{array}\right], \\
\mathbf{C}(\mathbf{p}) & =\left[\begin{array}{llll}
K_{0} p_{3}+K_{1} p_{1}+K_{2} p_{2} & -K_{1} p_{1} & -K_{2} p_{2}
\end{array}\right] .
\end{aligned}
$$

In most engineering applications, it is more plausible to use discrete time domain instead of continuous representations, due to the sampled nature of modern control systems. By introducing the discrete notation, at any time step $t$, one can rewrite (13) as:

$$
\left[\begin{array}{c}
\mathbf{x}_{t+1} \\
y_{t}
\end{array}\right]=\mathbf{S}(\mathbf{p})\left[\begin{array}{l}
\mathbf{x}_{t} \\
u_{t}
\end{array}\right]
$$

where the discretized system matrix, according to the zero order hold ( $\mathrm{ZOH})$ principle [28], can be written as

$$
\mathbf{S}(\mathbf{p})=\left[\begin{array}{cc}
T s \cdot \mathbf{A}(\mathbf{p})+\mathbf{I} & T s \cdot \mathbf{B} \\
\mathbf{C}(\mathbf{p}) & 0
\end{array}\right] .
$$

It is important to note that this is only an approximation of the original, continuous-time system, however, from the controller design point of view, more relevant for its better representation of digitally controlled robotic systems. $T s=1[\mathrm{~ms}]$ denotes the discrete time-step. This value was selected based on practical considerations, being a suitable processing time for current surgical systems. The domains were obtained by creating a rough estimate for the lower and upper limits of $x_{i}, i=1,2,3$ during manipulations. The MVS polytopic TP model form is written as:

$$
\begin{aligned}
& \mathbf{S}(\mathbf{p})=\mathcal{S} \underset{n=1}{\stackrel{3}{\otimes}} \mathbf{w}^{(n)}\left(p_{n, t}\right)= \\
& \quad=\mathcal{S} \times{ }_{1} \mathbf{w}^{(1)}\left(p_{1, t}\right) \times{ }_{2} \mathbf{w}^{(2)}\left(p_{2, t}\right) \times{ }_{3} \mathbf{w}^{(3)}\left(p_{3, t}\right)= \\
& =\sum_{j_{1}=1}^{2} \sum_{j_{2}=1}^{2} \sum_{j_{3}=1}^{2} w_{j_{1}}^{(1)}\left(p_{1}\right) w_{j_{2}}^{(2)}\left(p_{2}\right) w_{j_{3}}^{(3)}\left(p_{3}\right) \mathbf{S}_{j_{1}, j_{2}, j_{3}},
\end{aligned}
$$

where the core tensor $\mathcal{S}$ contains the $2 \times 2 \times 2$ vertexes and the corresponding univariate linear weighting functions, as shown in Fig. 2. 
TABLE I

PARAMETER ESTIMATION RESULTS FROM FORCE RELAXATION AND CONSTANT COMPRESSION RATE TESTS.

\begin{tabular}{|c|c|c|c|c|c|c|c||c|c|c|c|}
\hline $\begin{array}{c}K_{0} \\
{[N / m]}\end{array}$ & $\begin{array}{c}K_{1} \\
{[N / m]}\end{array}$ & $\begin{array}{c}K_{2} \\
{[N / m]}\end{array}$ & $\begin{array}{c}b_{1} \\
{[N s / m]}\end{array}$ & $\begin{array}{c}b_{2} \\
{[N s / m]}\end{array}$ & $\begin{array}{c}\kappa_{0} \\
{\left[m^{-1}\right]}\end{array}$ & $\begin{array}{c}\kappa_{1} \\
{\left[m^{-1}\right]}\end{array}$ & $\begin{array}{c}\kappa_{2} \\
{\left[m^{-1}\right]}\end{array}$ & $\begin{array}{c}p_{1} \\
{[-]}\end{array}$ & $\begin{array}{c}p_{2} \\
{[-]}\end{array}$ & $\begin{array}{c}p_{3} \\
{[-]}\end{array}$ & $\begin{array}{c}c_{0} \\
{[N / m]}\end{array}$ \\
\hline 2.03 & 0.438 & 0.102 & 5073 & 39.24 & 909.9 & 1522 & 81.18 & $0.9-213482$ & $0.9-2.10592$ & $0.9-1594.8$ & $1.9792-11000$ \\
\hline
\end{tabular}
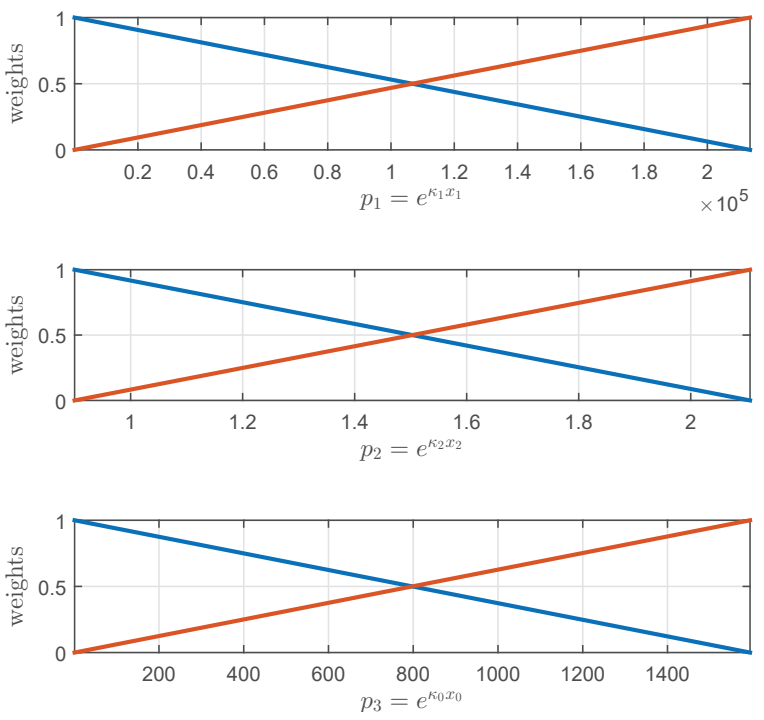

Fig. 2. Weighting functions of the MVS polytopic TP model presented by (13).

\section{Vi. Controller Design}

While (13) is mathematically suitable for stable statefeedback controller design, its practical realization is challenging due to the issue that the states $x_{1}$ and $x_{2}$ cannot be controlled directly, therefore their convergence to the desired $x_{i}=0$ state is very slow. On the other hand, $x_{0}$ can be affected directly, not taking the system dynamics into consideration, which subordinates the behavior to the dynamics of the relaxation poles. Therefore, achieving $x_{0}=0$ too soon would mean that the output of the system will only depend on the slowly converging states, which would not allow one to realize the desired force control performance in surgical robotics, in terms of speed and precision. To overcome these limitations, this paper proposes an alternative approach to the control problem, avoiding the setting of $x_{0}$ to a stationary state before the desired time. Let us consider the force output described in (11) the state of the system to be controlled. The derivative of expression (11) takes the form of

$$
\begin{aligned}
& \dot{F}=\dot{x}_{0} c_{0}\left(x_{0}, x_{1}, x_{2}\right)+ \\
& +\dot{x}_{1} c_{1}\left(x_{0}, x_{1}, x_{2}\right)+\dot{x}_{2} c_{2}\left(x_{0}, x_{1}, x_{2}\right),
\end{aligned}
$$

where

$$
\begin{array}{r}
c_{0}=K_{0} e^{\kappa_{0} x_{0}}\left(1+\kappa_{0} x_{0}\right)+K_{1} e^{\kappa_{1}\left(x_{0}-x_{1}\right)}\left(1+\kappa_{1}\left(x_{0}-x_{1}\right)\right)+ \\
+K_{2} e^{\kappa_{2}\left(x_{0}-x_{2}\right)}\left(1+\kappa_{2}\left(x_{0}-x_{2}\right)\right), \\
c_{1}=-K_{1} e^{\kappa_{1}\left(x_{0}-x_{1}\right)}\left(1+\kappa_{1} K_{1}\left(x_{0}-x_{1}\right)\right), \\
c_{2}=-K_{2} e^{\kappa_{2}\left(x_{0}-x_{2}\right)}\left(1+\kappa_{2} K_{2}\left(x_{0}-x_{2}\right)\right) .
\end{array}
$$

Let us consider

$$
\Delta F=F-F_{d},
$$

the new single state variable of the qLPV system, where $F_{d}$ is the desired reaction force to be achieved. The input of the system is $u=\dot{x}_{0}$, and the derivative of $\Delta F$ can be written as

$$
\frac{d}{d t} \Delta F=\dot{x}_{0} c_{0}+\dot{x}_{1} c_{1}+\dot{x}_{2} c_{2}-\dot{F}_{d} .
$$

In the equilibrium state, $\frac{d}{d t} \Delta F=0$, therefore

$$
u_{e q} c_{0}+\dot{x}_{1} c_{1}+\dot{x}_{2} c_{2}-\dot{F}_{d}=0,
$$

where $u_{e q}$ stands for the input at the equilibrium state. Following the idea on the error dynamics presented in Section V, the input of the second qLPV model can be introduced as:

$$
\Delta u=u-u_{e q},
$$

where

$$
u_{e q}=\frac{1}{c_{0}}\left(\dot{x}_{1} c_{1}+\dot{x}_{2} c_{2}-\dot{F}_{d}\right) .
$$

This approach allows us to collect all system variables and parameters in a single qLPV model parameter $c_{0}$, resulting in a very simple form. Introducing the time-discretization as discussed above, we can write:

$$
\Delta F_{t+1}=\Delta F_{t}+T s \cdot c_{0} \Delta u_{t} .
$$

The system matrix can be written in the form of:

$$
\mathbf{S}^{\prime}\left(c_{0}\right)=\left[\begin{array}{cc}
1 & T s \cdot c_{0} \\
1 & 0
\end{array}\right] .
$$

The core tensor $\mathcal{S}^{\prime}$ contains 2 vertexes

$$
\mathcal{S}_{(1)}^{\prime}=\left[\begin{array}{cc}
1 & 0.009 \\
1 & 0
\end{array}\right], \mathcal{S}_{(2)}^{\prime}=\left[\begin{array}{cc}
1 & 11 \\
1 & 0
\end{array}\right],
$$

the corresponding weighting functions are $\mathbf{w}^{\prime}$, as shown in Fig. 3. The parameter domain for $c_{0}$ was determined numerically, and was refined due to experimental considerations. The numerical values are listed in Table I.

The controller of the system is determined in the following form:

$$
\mathbf{u}=-\mathbf{F}(\mathbf{p}) \mathbf{x},
$$




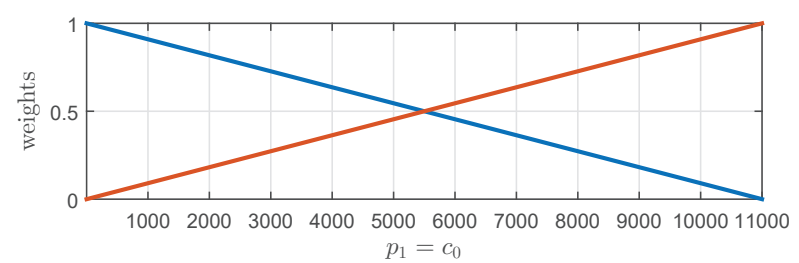

Fig. 3. Weighting function of the MVS polytopic TP model presented by (22).

where in this particular case:

$$
\mathbf{F}(\mathbf{p})=\mathcal{F} \underset{n=1}{\otimes} \mathbf{w}^{\prime}=\sum_{i=1}^{2} \mathcal{F}_{i} w_{i}^{\prime}\left(c_{0}\right),
$$

requiring a stable system in the Lyapunov sense. The final PDC (Parallel Distributed Compensator) controller was found solving the LQ optimal control problem using convex optimization algorithm provided by the Matlab tptool toolbox and the YALMIP interface [29], [30]. The resulting core tensor yields:

$$
\mathcal{F}=\left[\begin{array}{l}
0.36347 \\
0.08747
\end{array}\right] \text {. }
$$

The schematic block diagram of the controlled system is shown in Fig. 4.

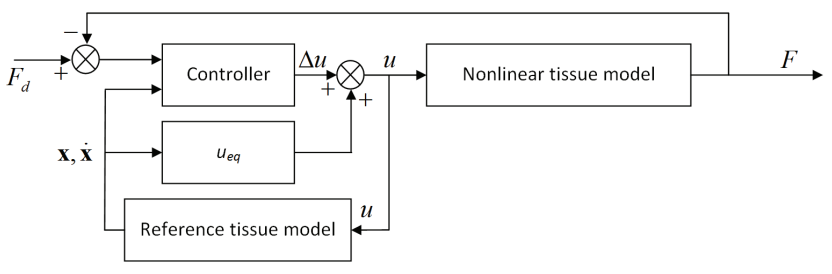

Fig. 4. Schematic block diagram of the controlled system.

\section{RESUlTS AND Discussion}

The proposed closed-loop controller solution was tested and simulated on a typical gesture of a surgical interventions. The process of grabbing, holding and releasing of the tissue was investigated by setting $F_{d}$ to a desired trajectory, followed by the investigation of control performance, addressing robustness. Three specific cases were investigated in the latter case: first, the real tissue parameters were ill-estimated, i.e., the reference tissue model parameters were $20 \%$ lower than the parameters used for controller design. Second, the simulation of a badly calibrated observer was done by linearly reducing the reference tissue model output by $20 \%$. Third, a time-delay term of $\tau=2[\mathrm{~ms}]$ was added to the reference tissue state output, modeling a slow observer behavior. Simulation results and the force tracking error for all cases are shown in Fig. 5, 6,7 and 8.

Fig. 5 shows that the proposed control scheme is suitable for realizing force control in a stable and precise manner, utilizing the selected soft tissue model. The tracking error for the

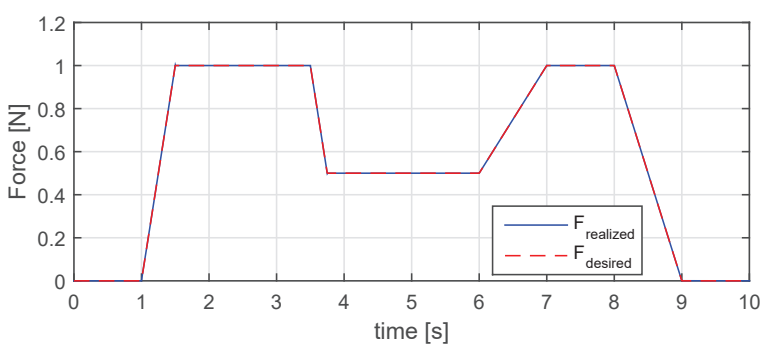

Fig. 5. Force tracking simulation results for modeling the grabbing, holding and release of the tissue. The simulation was carried out on the discrete time systems with the time-step of 1 [ms].

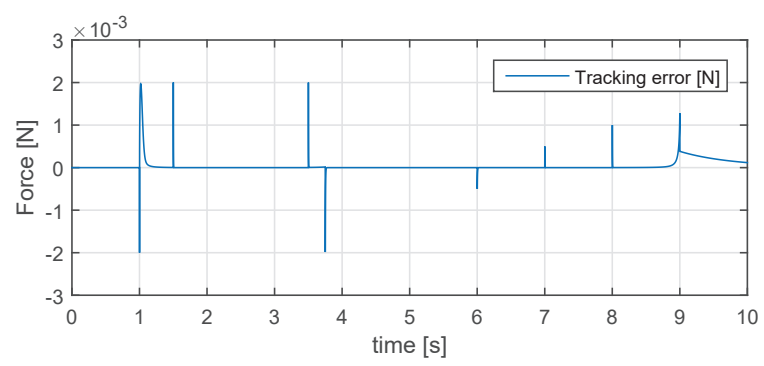

Fig. 6. Tracking error results for modeling the grabbing, holding and release of the tissue.

presented gesture did not exceed $5 \mathrm{mN}$, which is a favorably low value for surgical interventions. The results were achieved using the discrete sampling rate of $1 \mathrm{~ms}$, which is a realizable processing time for modern surgical systems in terms of arithmetic performance. The proposed controller was tested for robustness in the case of 3 different approaches, including ill-conditioned parameter estimation and observer design, and time-delay. The different behavior of these 3 cases is shown in Fig. 9, indicating that there is no significant decrease in the tracking performance under the mentioned disturbances. Minor oscillation can be observed in the case of delayed feedback, which, in when the delay time is increased, ultimately leads to stability loss. Further investigation of the phenomena and implementation of delay-based control schemes are part of our future work.

\section{CONCLUSION}

In this paper a control scheme and the corresponding control design methodology were presented for regulating interaction force during autonomous manipulation of soft biological tissues. The proposed approach utilizes recent results of polytopic model based control through the framework of Tensor Product Model Transformation. The goal of the presented control scheme is the regulation of reaction force during the robotic interaction with soft tissues e.g., grasp-hold-release cycles. Since biological tissues typically have highly nonlinear dynamic behavior (progressive stiffness characteristics, stress relaxation, etc.) time invariant linear controllers cannot provide ideal performance across the whole operation domain.

Based on our previously published nonlinear tissue model, the parameter-dependent error dynamics has been derived and 


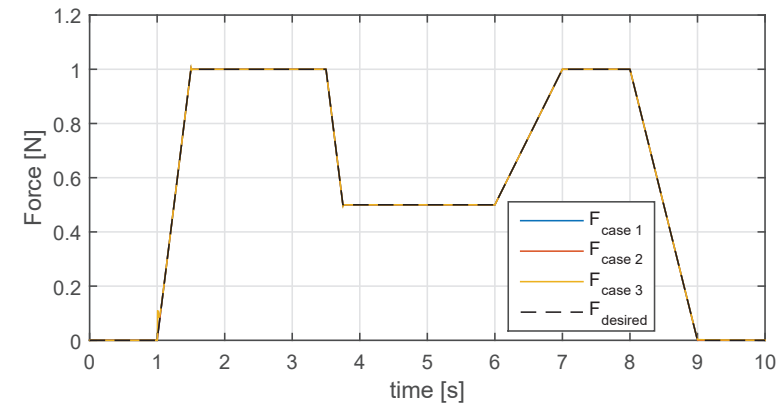

Fig. 7. Force tracking simulation results for modeling the grabbing, holding and release of the tissue, investigating the robustness of the proposed method. Case 1: incorrect estimation of the tissue parameters in the reference tissue model. Case 2: incorrectly calibrated observation, state output reduced by $20 \%$. Case 3: slow observation, state feedback is delayed by 2 [ms].
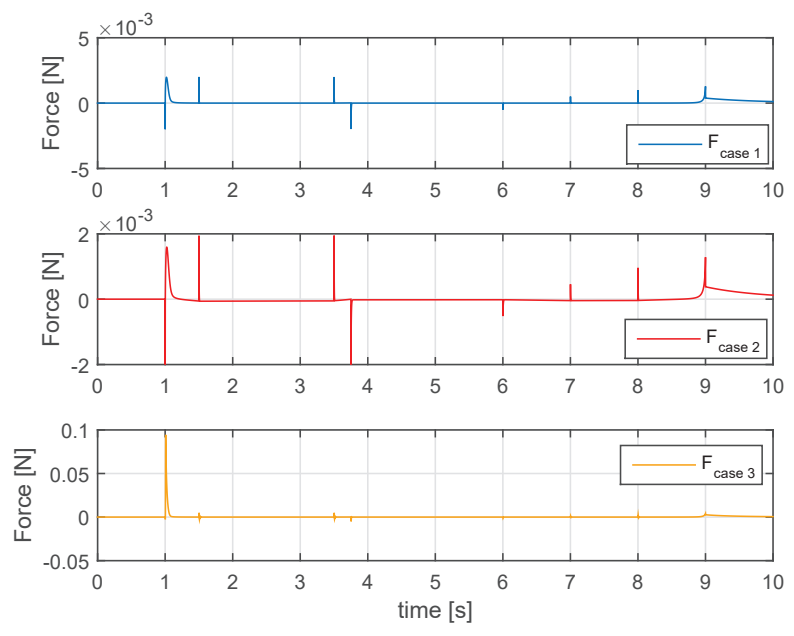

Fig. 8. Tracking error results for modeling the grabbing, holding and release of the tissue, investigating the robustness of the proposed method.

the resulted system has been reformulated in order to avoid the pitfall rendered by the slow dynamics of one state variable. The reformulated system allows for concentrating the three original parameter dependencies into a single parameter and construct a feed forward term for the equilibrial input. An additional state feedback controller was utilized that handle the unmodeled dynamics and further disturbances. Since the state variables cannot be measured in the real process, a reference tissue model has been used. The state feedback controller was designed by LMI-based synthesis providing the variable gains as parameter dependent polytopic TP functions. The overall system has been evaluated via numerical simulations, with very promising results. The implementation of the proposed method into supervised telemanipulation/telesurgical equipments and into surgical, invasive intervention and virtual trainers would enhance the performance of these systems, allowing haptic sensing to the operator. Our future work includes the experimental validation of the system in both virtual and ex vivo environments, extending the model with a discrete-time PDC state observer.

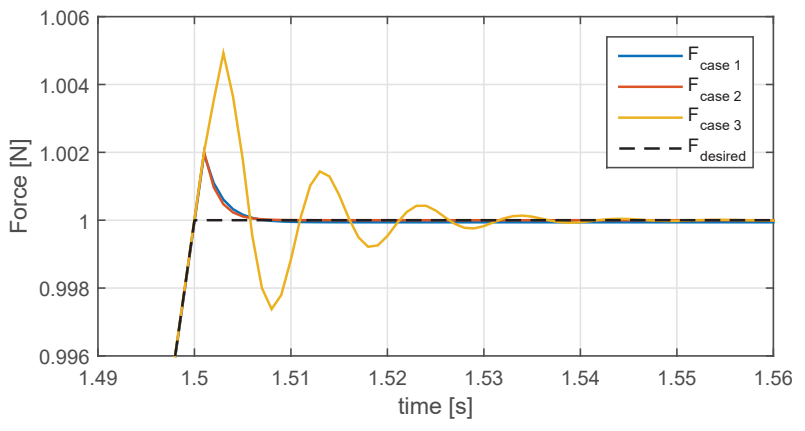

Fig. 9. Tracking performance in the most critical point of the simulation according to the tracking error results.

\section{ACKNOWLEDGMENT}

The research was supported by the Ministry of Human Capacities of Hungary. Tamás Haidegger is a Bolyai Fellow of the Hungarian Academy of Sciences. This work has been supported by ACMIT (Austrian Center for Medical Innovation and Technology), which is funded within the scope of the COMET (Competence Centers for Excellent Technologies) program of the Austrian Government. The research was supported by the Hungarian OTKA PD 116121 grant.

\section{REFERENCES}

[1] Á. Takács, D. A. Nagy, I. J. Rudas, and T. Haidegger, "Origins of surgical robotics: From space to the operating room," Acta Polytechnica Hungarica, vol. 13, no. 1, 2016.

[2] T. Haidegger, B. Benyó, L. Kovács, and Z. Benyó, "Force sensing and force control for surgical robots," in 7th IFAC Symposium on Modeling and Control in Biomedical Systems, vol. 7, no. 1, 2009.

[3] Á. Takács, L. Kovács, I. J. Rudas, R.-E. Precup, and T. Haidegger, "Models for force control in telesurgical robot systems," Acta Polytechnica Hungarica, vol. 12, no. 8, pp. 95-114, 2015.

[4] Á. Takács, S. Jordán, R.-E. Precup, L. Kovács, J. Tar, I. Rudas, and T. Haidegger, "Review of tool-tissue interaction models for robotic surgery applications," in 2014 IEEE 12th International Symposium on Applied Machine Intelligence and Informatics (SAMI), 2014, pp. 339344.

[5] P. Kazanzides, J. Zuhars, B. Mittelstadt, and R. H. Taylor, "Force sensing and control for a surgical robot," in IEEE International Conference on Robotics and Automation, 1992, pp. 612-617.

[6] M. C. Lee, C. Y. Kim, B. Yao, W. J. Peine, and Y. E. Song, "Reaction force estimation of surgical robot instrument using perturbation observer with smcspo algorithm," in Advanced Intelligent Mechatronics (AIM), 2010 IEEE/ASME International Conference on. IEEE, 2010, pp. 181186.

[7] S. G. Yuen, D. P. Perrin, N. V. Vasilyev, P. J. Del Nido, and R. D. Howe, "Force tracking with feed-forward motion estimation for beating heart surgery," Robotics, IEEE Transactions on, vol. 26, no. 5, pp. 888-896, 2010.

[8] C. Liu, P. Moreira, N. Zemiti, and P. Poignet, "3d force control for robotic-assisted beating heart surgery based on viscoelastic tissue model," in Engineering in Medicine and Biology Society, EMBC, 2011 Annual International Conference of the IEEE. IEEE, 2011, pp. 70547058.

[9] P. Moreira, C. Liu, N. Zemiti, and P. Poignet, "Soft tissue force control using active observers and viscoelastic interaction model," in Robotics and Automation (ICRA), 2012 IEEE International Conference on. IEEE, 2012, pp. $4660-4666$.

[10] S. B. Kesner and R. D. Howe, "Robotic catheter cardiac ablation combining ultrasound guidance and force control," The International Journal of Robotics Research, vol. 33, no. 4, pp. 631-644, 2014. 
[11] N. Zemiti, G. Morel, T. Ortmaier, and N. Bonnet, "Mechatronic design of a new robot for force control in minimally invasive surgery," Mechatronics, IEEE/ASME Transactions on, vol. 12, no. 2, pp. 143-153, 2007.

[12] A. Takács, P. Galambos, P. Pausits, and T. Haidegger, "Nonlinear Soft Tissue Models and Force Control for Medical Cyber-Physical Systems," in Proceddings of the 2015 IEEE International Conference on Systems, Man, and Cybernetics. Hong Kong: IEEE, Oct. 2015, pp. 1520-1525.

[13] A. Takács, T. Haidegger, P. Galambos, J. Kuti, and I. Rudas, "Nonlinear soft tissue mechanics based on polytopic tensor product modeling," in 2016 IEEE 14th International Symposium on Applied Machine Intelligence and Informatics (SAMI), 2016, pp. 211-215.

[14] P. Baranyi, Y. Yam, and P. Várlaki, Tensor Product Model Transformation in Polytopic Model-Based Control, 1st ed. Boca Raton: CRC Press, 2013.

[15] P. Baranyi, "TP model transformation as a way to LMI-based controller design," IEEE Transactions on Industrial Electronics, vol. 51, no. 2, pp. 387-400, 2004.

[16] L. D. Lathauwer, B. D. Moor, and J. Vandewalle, "A multilinear singular value decomposition," SIAM Journal on Matrix Analysis and Applications, vol. 21, no. 4, pp. 1253-1278, 2000.

[17] P. Grof and Y. Yam, "Furuta Pendulum A Tensor Product Model-Based Design Approach Case Study," in 2015 IEEE International Conference on Systems, Man, and Cybernetics (SMC), Oct. 2015, pp. 2620-2625.

[18] J. Kuti, P. Galambos, and A. Miklós, "Output Feedback Control of a Dual-Excenter Vibration Actuator via qLPV Model and TP Model Transformation: Control Design of a Dual-excenter Vibration Actuator," Asian Journal of Control, vol. 17, no. 2, pp. 432-442, 2015.

[19] P. Galambos, P. Baranyi, and G. Arz, "Tensor product model transformation-based control design for force reflecting tele-grasping under time delay," Proceedings of the Institution of Mechanical Engineers, Part C: Journal of Mechanical Engineering Science, vol. 228, no. 4, pp. 765-777, Mar. 2014.

[20] P. Galambos, J. Kuti, P. Baranyi, and I. J. Rudas, "Tensor Product based
Convex Polytopic Modeling of Nonlinear Insulin-Glucose Dynamics," in Proceddings of the 2015 IEEE International Conference on Systems, Man, and Cybernetics. Hong Kong: IEEE, Oct. 2015, pp. 2597-2602.

[21] M. Hoeckelmann, I. J. Rudas, P. Fiorini, F. Kirchner, and T. Haidegger, "Current capabilities and development potential in surgical robotics," Int J Adv Robot Syst, vol. 12, p. 61, 2015.

[22] T. Haidegger, L. Kovács, R.-E. Precup, B. Benyó, Z. Beny, and S. Preitl, "Simulation and control for telerobots in space medicine," Acta Astronautica, vol. 81, no. 1, pp. 390-402, Dec. 2012

[23] J. Rosen, J. D. Brown, S. De, M. Sinanan, and B. Hannaford, "Biomechanical properties of abdominal organs in vivo and postmortem under compression loads," Journal of Biomechanical Engineering, vol. 130, no. 2, p. $021020,2008$.

[24] Y. Bao, D. Wu, Z. Yan, and Z. Du, "A new hybrid viscoelastic soft tissue model based on meshless method for haptic surgical simulation," The open biomedical engineering journal, vol. 7, p. 116, 2013.

[25] T. Yamamoto, Applying tissue models in teleoperated robot-assisted surgery. The Johns Hopkins University, 2011.

[26] K. L. Troyer, S. S. Shetye, and C. M. Puttlitz, "Experimental characterization and finite element implementation of soft tissue nonlinear viscoelasticity," Journal of biomechanical engineering, vol. 134, no. 11, p. 114501, 2012.

[27] Á. Takács, I. J. Rudas, and T. Haidegger, "Surface deformation and reaction force estimation of liver tissue based on a novel nonlinear massspring-damper viscoelastic model," Medical \& biological engineering \& computing, pp. 1-10, 2015.

[28] G. F. Franklin, J. D. Powell, and M. L. Workman, Digital control of dynamic systems. Addison-wesley Menlo Park, 1998, vol. 3.

[29] J. Lofberg, "YALMIP: A toolbox for modeling and optimization in MATLAB," in Computer Aided Control Systems Design, 2004 IEEE International Symposium on. IEEE, 2004, pp. 284-289.

[30] E. Andersen, B. Jensen, J. Jensen, R. Sandvik, and U. Worsøe, "Mosek version 6," Technical Report TR-2009-3, MOSEK, Tech. Rep., 2009. 